\title{
Article
}

\section{Combined in vitro and in silico approaches to the assessment of stimulant properties of novel psychoactive substances - The case of the benzofuran 5-MAPB}

Sahai, Michelle, Davidson, Colin, Khelashvili, George, Barrese, Vincenzo, Dutta, Neelakshi, Weinstein, Harel and Opacka-Juffry, Jolanta

Available at https://clok.uclan.ac.uk/16476/

Sahai, Michelle, Davidson, Colin orcid iconORCID: 0000-0002-8180-7943, Khelashvili, George, Barrese, Vincenzo, Dutta, Neelakshi, Weinstein, Harel and Opacka-Juffry, Jolanta (2016) Combined in vitro and in silico approaches to the assessment of stimulant properties of novel psychoactive substances - The case of the benzofuran 5-MAPB. Progress in Neuro psychopharmacology and Biological Psychiatry, 75 . pp. 1-9. ISSN 0278-5846

It is advisable to refer to the publisher's version if you intend to cite from the work. http://dx.doi.org/10.1016/j.pnpbp.2016.11.004

For more information about UCLan's research in this area go to http://www.uclan.ac.uk/researchgroups/ and search for <name of research Group>.

For information about Research generally at UCLan please go to http://www.uclan.ac.uk/research/

All outputs in CLoK are protected by Intellectual Property Rights law, including Copyright law. Copyright, IPR and Moral Rights for the works on this site are retained by the individual authors and/or other copyright owners. Terms and conditions for use of this material are defined in the policies page. 


\section{Accepted Manuscript}

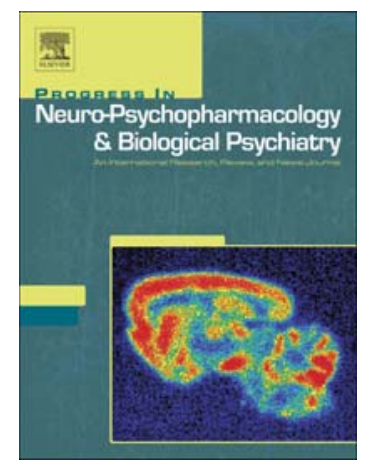

Combined in vitro and in silico approaches to the assessment of stimulant properties of novel psychoactive substances - The case of the benzofuran 5-MAPB

Michelle A. Sahai, Colin Davidson, George Khelashvili, Vincenzo Barrese, Neelakshi Dutta, Harel Weinstein, Jolanta Opacka-Juffry

PII: $\quad$ S0278-5846(16)30397-9

DOI: $\quad$ doi: $10.1016 /$ j.pnpbp.2016.11.004

Reference: $\quad$ PNP 8982

To appear in: $\quad$ Progress in Neuropsychopharmacology \& Biological Psychiatry

Received date: 28 July 2016

Revised date: $\quad 19$ November 2016

Accepted date: 21 November 2016

Please cite this article as: Sahai Michelle A., Davidson Colin, Khelashvili George, Barrese Vincenzo, Dutta Neelakshi, Weinstein Harel, Opacka-Juffry Jolanta, Combined in vitro and in silico approaches to the assessment of stimulant properties of novel psychoactive substances - The case of the benzofuran 5-MAPB, Progress in Neuropsychopharmacology \& Biological Psychiatry (2016), doi: 10.1016/j.pnpbp.2016.11.004

This is a PDF file of an unedited manuscript that has been accepted for publication. As a service to our customers we are providing this early version of the manuscript. The manuscript will undergo copyediting, typesetting, and review of the resulting proof before it is published in its final form. Please note that during the production process errors may be discovered which could affect the content, and all legal disclaimers that apply to the journal pertain. 
Combined in vitro and in silico approaches to the assessment of stimulant properties of novel psychoactive substances - the case of the benzofuran 5-MAPB

Michelle A. Sahai ${ }^{1 *}$, Colin Davidson ${ }^{2,3^{*}}$, George Khelashvili ${ }^{4}$, Vincenzo Barrese ${ }^{2}$, Neelakshi Dutta $^{2}$, Harel Weinstein ${ }^{4,5}$ and Jolanta Opacka-Juffry ${ }^{1}$

${ }^{1}$ University of Roehampton, London SW15 4JD, UK

${ }^{2}$ St George's University of London, Cranmer Terrace, London SW17 0RE, UK

${ }^{3}$ Pharmacy \& Biomedical Sciences, University of Central Lancashire, Preston PR1 2HE, UK

${ }^{4}$ Department of Physiology and Biophysics, Weill Cornell Medical College of Cornell University (WCMC), New York, NY, 10065, USA

${ }^{5} \mathrm{HRH}$ Prince Alwaleed Bin Talal Bin Abdulaziz Alsaud Institute of Computational Biomedicine, Weill Cornell Medical College of Cornell University, New York, NY, 10065, USA

*Joint first authors

Correspondence: Prof. J. Opacka-Juffry, Department of Life Sciences, University of Roehampton, London SW15 4JD, UK

Tel +44(0)20 83923563

Email: j.opacka-juffry@ roehampton.ac.uk 


\begin{abstract}
Novel psychoactive substances (NPS) are increasingly prevalent world-wide although their pharmacological characteristics are largely unknown; those with stimulant properties, due to interactions with the dopamine transporter (DAT), have addictive potential which their users may not realise.
\end{abstract}

We evaluated the binding of 1-(1-benzofuran-5-yl)-N-methylpropan-2-amine (5-MAPB) to rat striatal DAT by means of quantitative autoradiography with $\left[{ }^{125}\right.$ I]RTI-121, and the effects of 5-MAPB on electrically-evoked dopamine efflux by fast-cyclic voltammetry in rat brain slices. 5-MAPB displaced $\left[{ }^{125} \mathrm{I}\right] \mathrm{RTI}-121$ in a concentration-dependent manner, with significant effects at 10 and $30 \mu \mathrm{M}$. The voltammetry data suggest that 5-MAPB reduces the rate of dopamine reuptake; while the peak dopamine efflux was not increased, the area under the curve was augmented. 5-MAPB can also cause reverse dopamine transport consistent with stimulant properties, more similar to amphetamine than cocaine. Molecular modelling and docking studies compared the binding site of DAT in complex with 5-MAPB to dopamine, amphetamine, 5-APB, MDMA, cocaine and RTI-121. This structural comparison reveals a binding mode for 5-MAPB found in the primary binding (S1) site, central to transmembrane domains 1, 3, 6 and 8, which overlaps with the binding modes of dopamine, cocaine and its analogues. Atomistic molecular dynamics simulations further show that, when in complex with 5-MAPB, DAT can exhibit conformational transitions that spontaneously isomerize the transporter into inward-facing state, similarly to that observed in dopaminebound DAT.

These novel insights, offered by the combination of computational methods of biophysics with neurobiological procedures, provide structural context for NPS at DAT and relate them with their functional properties at DAT as the molecular target of stimulants. 
Key words: addiction, autoradiography, dopamine transporter, legal highs, molecular modelling, voltammetry 


\section{Introduction}

Among novel psychoactive substances (NPS), benzofurans have a prominent presence in Europe and elsewhere. They are phenethylamine derivatives and related to methylenedioxyphenethylamines such as 3,4-methylenedioxy-methamphetamine (MDMA, Ecstasy) and 3,4-methylenedioxy-amphetamine (MDA), both classified as Class A drugs in the U.K. Benzofuran analogues of MDA were first synthesised in the early 1990s (Monte et al., 1993) and became prevalent as recreational drugs, also known as 'legal highs', nearly two decades later. They were permanently banned as Class B substances in the U.K. in 2014 (The Misuse of Drugs Act 1971 (Ketamine etc.) (Amendment) Order 2014, n.d.).

Benzofurans, similarly to MDA and MDMA, evoke both empathogenic (entactogenic) and stimulant effects in users, according to anecdotal reports. While entactogenic properties are those which users value for the type of drug-related experience, the stimulant element associates with addiction liability that the user may not realise. Stimulant drugs normally increase dopamine availability in the brain, including the dorsal and ventral striatum, the latter being part of the mesolimbic pathway implicated in the reward-related behaviour (Di Chiara and Bassareo, 2007). It has been convincingly demonstrated that dopamine release in the nucleus accumbens of this pathway associates with the perception of pleasure and reward; it also contributes to the biological underpinnings of drug dependence (Di Chiara and Imperato, 1988). The dopamine transporter (DAT) is the molecular target for stimulants, responsible for their dopamine-enhancing effects, as originally proposed for cocaine that binds at DAT and inhibits dopamine re-uptake thus potentiating dopaminergic neurotransmission in mesolimbic/mesocortical pathways (Kuhar et al., 1991).

We have previously examined a benzofuran, 5-APB (5-(2-aminopropyl)benzofuran, 1benzofuran-5-ylpropan-2-amine, benzofury), and found it to bind to the DAT as shown 
through a displacement of $\left[{ }^{125}\right.$ I]RTI-121 from the DAT in the striatal and cortical areas of rat brain, apart from its activity at 5- $\mathrm{HT}_{2 \mathrm{~A}}$ receptors (Dawson et al., 2014). 5-APB also caused an increase in electrically evoked dopamine efflux, and at high concentrations caused reverse transport of dopamine in rat accumbens brain slices (Dawson et al., 2014). Another study using cell lines has demonstrated that 5-APB binds to other members of the neurotransmitter: sodium symporter (NSS) family of monoamine transporters, with greater affinity at the DAT $>$ serotonin transporter $($ SERT) $>$ norepinephrine transporter (NET) (Iversen et al., 2013). In contrast, 5-APB has been found to have a higher preference to the SERT than DAT in human embryonic kidney cells (Rickli et al., 2015). The pKi of 5-APB and MDMA at human cloned monoamine transporters have been determined: 5-APB: 6.33; 6.30; 5.78, (-)MDMA: 5.50; 5.21; 5.17, (+)MDMA: 5.70; 5.62; 5.56 for the NET, DAT and SERT respectively (Iversen et al., 2013). To our knowledge the pKi for 5-MAPB at monoamine transporters has not yet been determined although this drug has been shown to displace monoamine radioligands: at 10 $\mu \mathrm{M}$ 5-MAPB displaced 33\% 3H-nisoxetine (NET), 73\% 3H-BTCP (DAT) and 75\% 3Himipramine (SERT) (Shimshoni et al., 2016).

For the present study, we have chosen 1-(1-benzofuran-5-yl)-N-methylpropan-2-amine (5MAPB) which is a N-methylated version of 5-APB, structurally related to MDMA (Welter et al., 2015a, 2015b). Online drug user fora suggest that 5-MAPB, despite its illegality, is still being used today. Its psychoactive effects include euphoria, visual distortions and reduced inhibitions. Inexperienced users appear to feel that 5-MAPB is similar to MDMA, while experienced users feel that it is quite unique, with less empathy than one might associate with MDMA and reduced sexual dysfunction versus MDMA. Unwanted effects include hyperthermia, tachycardia, restlessness, inability to sleep, nystagmus, pupil dilation and jaw tension. Some users suggest taking 5-MAPB with a psychedelic dissociative or alcohol to enhance the psychoactive effects, or a benzodiazepine to reduce unwanted side-effects and 
help with sleep (Erowid; Bluelight; The Trip Report). On firmer scientific grounds, the National Program on Substance Abuse Deaths (np_SAD report, 2014) in the UK has reported nine instances of benzofurans found in post mortem toxicology in the UK in 2012 (the latest figures) with benzofurans being cited as the actual cause of death in seven of these cases (np_SAD report, 2014).

Here, we aim to assess the stimulant properties of 5-MAPB through in vitro and in silico tests that include the evaluation of drug binding to rat striatal DAT by means of quantitative autoradiography with $\left[{ }^{125}\right.$ I]RTI-121 as a selective DAT ligand (Hume et al., 1996). We also use a functional test measuring electrically evoked dopamine efflux in the nucleus accumbens, as the brain region involved in reinforcement and addiction, by means of fast cycling voltammetry. We augment our ligand binding and functional data with structural results from computational molecular modelling, docking studies and atomistic molecular dynamics simulations, which have been previously shown to be robust methods for predicting the binding sites of various psychostimulants at human DAT (hDAT) in relation to the substrate binding site (Beuming et al., 2008; Bisgaard et al., 2011). We present here, on the basis of the crystal structure from Drosophila melanogaster dopamine transporter (dDAT) (PDB ID: 4XP1) (Wang et al., 2015), a molecular model of 5-MAPB bound to rat DAT (rDAT), in comparison with that of dopamine, amphetamine, 5-APB, MDMA, cocaine and RTI-121 also bound to rDAT. Our studies compare the structural changes produced by various ligands binding to rDAT to the already ongoing work in hDAT models (Hamilton et al., 2013; Hansen et al., 2014; Khelashvili et al., 2015a, 2015b) since it is the primary animal model used in functional studies and pre-clinical drug abuse testing.

We propose here that such a structural analysis is important when creating a stimulant profile of NPS as it adds to the insights of binding and functional data derived from the so-far used 
neurobiological techniques, autoradiography and voltammetry, applied in animal models. It is important to understand the molecular determinants of stimulant NPS actions, which may underlie their distinct pharmacological effects at DAT. 


\section{Methods}

2.1 Animals. Eight week old male Wistar rats (Charles River, UK) were kept on a 12/12 h light/dark cycle (lights on at $7 \mathrm{AM}$ ). Food and water were freely available and rats were housed 6 per cage. Temperature and humidity were $18-22^{\circ} \mathrm{C}$ and $55 \pm 15 \%$ respectively. Rats were treated in accordance with the U.K. Animals (Scientific Procedures) Act 1986 (related to the 1986 EU Directive 86/609/EEC) and sacrificed by cervical dislocation with no anaesthesia. In the ligand binding studies we used 6 rats. For the voltammetry studies we used approximately 20 rats to ensure that each concentration of 5-MAPB was tested in a bran slice from a different rat.

2.2 Chemicals. All chemicals used were supplied by Sigma Chemicals (Poole, UK) except 5MAPB which was a gift from John Ramsey (TICTAC Communications Ltd, UK). The radioligand for the dopamine transporter, $\left[{ }^{125} \mathrm{I}\right] \mathrm{RTI}-121$ (specific activity $81.4 \mathrm{TBq} / \mathrm{mmol}$ ) was purchased from Perkin Elmer (Beaconsfield, U.K.).

2.3 Radioligand binding study at the dopamine transporter. Brains were removed and frozen at $-40^{\circ} \mathrm{C}$ in a mixture of methanol and dry ice, then stored at $-80^{\circ} \mathrm{C}$. Frozen brains were cut into $20 \mu \mathrm{m}$ coronal sections to harvest the striatum at $+1.7 \mathrm{~mm}$ to $-0.3 \mathrm{~mm}$ versus bregma (Paxinos, G Watson, 2007). Serial striatal sections were collected onto polysinecoated slides and stored at $-80^{\circ} \mathrm{C}$ prior to autoradiography. The autoradiography procedure was conducted according to Strazielle et al., 1998. After preincubation in $0.05 \mathrm{M} \mathrm{NaPB} \mathrm{pH}$ 7.4, sections were incubated with $20 \mathrm{pM}\left[{ }^{125} \mathrm{I}\right] \mathrm{RTI}-121$ in $\mathrm{NaPB} \mathrm{pH} 7.4$ with increasing concentrations of 5-MAPB $(0-30 \mu \mathrm{M})$ for $60 \mathrm{~min}$ at room temperature. Non-specific binding was assessed in the presence of $200 \mu \mathrm{M}$ nomifensine ('block' - control). Rinsed and air-dried slides were apposed to Kodak BioMax MR films for 3 days; autoradiograms were analysed using $\mathrm{MCID}^{\mathrm{TM}}$, Version 7.0, Imaging Research Inc., Interfocus Ltd, U.K. Flat-field correction was applied. The caudate regions of interest were sampled in duplicates for 
relative optical density; left and right caudate values were averaged and their means were calculated to assess the specific binding.

2.4 Brain slice preparation for functional neurochemistry studies. The brain was removed and cut to produce a block containing the accumbens $(-5$ to $+3 \mathrm{~mm}$ Vs bregma (Paxinos, $\mathrm{G}$ Watson, 2007)) which was attached to the chuck of a vibratome, using cyanoacrylate glue, then immersed in ice-cold artificial cerebro-spinal fluid (aCSF; $\mathrm{pH}$ 7.4). Coronal slices (400 $\mu \mathrm{m})$ at the level of the accumbens $(+1.0$ to $+2.2 \mathrm{~mm}$ versus bregma) were transferred to a slice saver where the slices were immersed in aCSF continuously bubbled with $95 \% \mathrm{O}_{2} / 5 \%$ $\mathrm{CO}_{2}$ at room temperature $\left(22 \pm 1^{\circ} \mathrm{C}\right)$. After at least $60 \mathrm{~min}$, slices were transferred to the brain slice chamber, which was heated by a circulating water bath (Grant, Cambridgeshire, UK) and continuously perfused with aCSF $(100 \mathrm{ml} / \mathrm{h})$. The temperature in the brain slice chamber was $32.5 \pm 0.5^{\circ} \mathrm{C}$ and the slice was equilibrated for $30 \mathrm{~min}$ prior to electrical stimulation. The composition of aCSF was: (mM): $\mathrm{NaCl}$ (126.0), $\mathrm{KCl}(2.0), \mathrm{KH}_{2} \mathrm{PO}_{4}(1.4)$, $\mathrm{MgSO}_{4}$ (2.0), $\mathrm{NaHCO}_{3}(26.0), \mathrm{CaCl}_{2}$ (2.4), (+)glucose (10.0), bubbled for at least $60 \mathrm{~min}$ with $95 \% \mathrm{O}_{2} / 5 \% \mathrm{CO}_{2}$.

2.5 Fast cyclic voltammetry (FCV). Carbon fibre electrodes were fabricated by sucking a 7 $\mu \mathrm{m}$ diameter carbon fibre into a $10 \mathrm{~cm}$ length borosilicate glass capillary (o.d., $2.0 \mathrm{~mm}$; i.d., $1.16 \mathrm{~mm}$ : Harvard Apparatus LTD, Kent, UK) using a vacuum. This was then pulled to a fine tip (Model P-30, Sutter Instruments Co, USA). The carbon fibre protruding from the pulled tip was then cut to a length of $\sim 75 \mu \mathrm{m}$ using a scalpel, under microscopic guidance.

Triangular voltammetric scans ( 0 to -1.0 to +1.4 to -1 and back to $0 \mathrm{~V}$ vs. $\mathrm{Ag} / \mathrm{AgCl}, 480 \mathrm{~V} / \mathrm{s}$; $8 \mathrm{~Hz}$ ) were applied to the carbon electrode using a Millar Voltammetric Analyser (PD Systems, West Molesey, UK), with complete voltammetric scans recorded digitally at 40,000 Hz (CED1401 Micro3; Spike2 v7.06 software Cambridge Electronic Design, UK). An 
increase in the current signal at $\sim 600 \mathrm{mV}$, together with a corresponding reduction peak at $200 \mathrm{mV}$, are characteristic of dopamine detection in the accumbens (Robinson et al., 2003). See Figure 1.

[Figure 1 here]

At the start of the 30 min equilibration period, the tip of the carbon electrode was placed into the accumbens core, at a depth of approximately $75 \mu \mathrm{m}$, using a micromanipulator and under microscope guidance. This allowed us to monitor the stability of the brain slice as on some occasions (e.g. poor slice health) the slice can release large amounts of dopamine (Davidson et al., 2011). A bipolar stimulating electrode, made from 2 stainless steel sharp-tipped electrodes (Plastics One, VA USA) was placed about $200 \mu \mathrm{m}$ away from the carbon electrode with the tips inserted into the slice to $\sim 100 \mu \mathrm{m}$ using a micromanipulator. Auxiliary (stainless steel) and reference $(\mathrm{Ag} / \mathrm{AgCl})$ electrodes were placed in 2 corners of the bath.

2.5.1 Stimulation procedure. We used bipolar electrodes, tips $\approx 500 \mu \mathrm{m}$ apart to locally stimulate the core of the accumbens. Pseudo-one pulse stimulation (Singer, 1988) was used in order to avoid autoreceptor activation, which occurs at about $500 \mathrm{~ms}$ after striatal dopamine release (Lee et al., 2002; Phillips et al., 2002). The stimulus train was 10 x 1 ms pulses $10 \mathrm{~ms}$ apart $(100 \mathrm{~Hz})$ with a constant current of $10 \mathrm{~mA}$ using a Neurolog NL800 stimulus isolator. Pulses were programmed using the CED Spike software.

2.5.2 FCV data analysis. Carbon electrodes were calibrated in 5-10 $\mu \mathrm{M}$ dopamine and the brain slice signals were then converted to dopamine concentrations. We measured the area under the curve (AUC) of dopamine efflux (Yorgason et al., 2011). We also noted changes in 
basal levels of dopamine, which would suggest reverse transport of dopamine, as seen with amphetamine-like compounds.

2.6 Homology model of rDAT in complex with dopamine. The construction of the homology model of rDAT ( $\mathrm{rDAT}^{\mathrm{dDAT}}$ ) in complex with dopamine uses established protocols previously described for the construction of a human DAT (hDAT) model (Hamilton et al., 2013; Hansen et al., 2014; Khelashvili et al., 2015a, 2015b). Briefly, as the template, the homology model uses the recent crystal structure of the neurotransmitter sodium symporter (NSS) DAT from Drosophila melanogaster (dDAT) bound to dopamine (PDB ID: 4XP1) (Wang et al., 2015). The model of $\mathrm{rDAT}^{\mathrm{dDAT}}$ was then prepared with the Protein Preparation Wizard of Schrödinger, using a standard protocol with the advanced OPLS3 force field implemented in Release 2016-2 ('Schrödinger, LLC: Portland, OR, 2007, Web address: www.schrodinger.com.', n.d.). Dopamine was then re-docked into this prepared model of rDAT $^{\mathrm{dDAT}}$ as described earlier (Khelashvili et al., 2015a, 2015b).

The rDAT $^{\mathrm{dDAT}}$ homology model was used in all docking studies after an extensive comparison was done to compare the critical transmembrane regions for all fourteen dDAT crystal structures. As can be seen from Supplemental Table 1 the Root Mean Square Deviation (RMSD) between dDAT crystal structure (PDB ID: 4XP1) and all other thirteen dDAT crystal structures (bound to either antidepressants, psychostimulants or mutant dDAT bound to psychostimulants) are negligible, especially with a RMSD of $<1 \AA$ for the critical regions of the substrate dopamine binding site and ion binding sites, TMs $1,6 \& 8$, which are key to the inferences we describe herein. 


\subsection{Binding models of 5-MAPB, amphetamine, 5-APB, MDMA, cocaine and RTI-121.}

The chemical structure of 5-MAPB was built using the LigPrep module of Schrödinger Release 2016-2. 5-MAPB was subsequently docked into the rDAT model using the following steps implemented in the Induced fit docking (IFD) protocol in the Schrödinger software suite: (i) The receptor grid was defined as an enclosing box at the centroid of selected residues within $4 \AA$ of dopamine; (ii) In the initial Glide docking stage, a soft potential docking with van der Waals radii scaling of 0.5 for the protein and ligand was performed retaining a maximum number of 20 poses per ligand; (iii) Residues within $7.0 \AA$ of ligand poses were kept free to move in the Prime refinement step, and the side chains were further optimized; (iv) Poses within $30 \mathrm{kcal} / \mathrm{mol}$ of the lowest energy structure obtained in the previous step were re-docked using Glide XP; (v) The binding energy (IFDScore) for each output pose was computed as implemented in the IFD protocol. This score is the sum of the GlideScore from the redocking step and 5\% of the Prime energy from the refinement calculation. The most favorable binding conformation of the 5-MAPB-rDAT complex (entry 6 in Supplemental Figure 1SA and 1SC) was selected for analysis, based on the IFDScore and visual inspection that coincides with the expectations from the dDAT crystal structures with bound dopamine, cocaine and amphetamine and other compounds (Beuming et al., 2006). See Supplemental Figure 1S for details. The binding poses for 5-APB and MDMA in rDAT $^{\mathrm{dDAT}}$ were determined using a similar protocol.

The chemical structures of cocaine and amphetamine were extracted from the crystallographic structures of dDAT (Wang et al., 2015); hydrogen atoms were added and the most favorable protonation state was calculated with Protein Preparation Wizard. The cocaine scaffold was manipulated in the Build module of Schrödinger Release 2016-2, to add and remove atoms to produce RTI-121, followed by the same treatment in the Protein Preparation Wizard to calculate the most favorable protonation state. DDAT $^{\mathrm{dDAT}}$ complexes bound to 
cocaine, amphetamine and RTI-121 were prepared using established protocols (Khelashvili et al., 2015a, 2015b).

2.8 Molecular dynamics simulations: The previously well-equilibrated structure of the occluded hDAT ${ }^{\mathrm{dDAT}}$ model (homology model of the full-length hDAT based on dDAT X-ray structure 4M48 with added N- and C-terminal domains) in complex with dopamine and in a corresponding membrane environment (Khelashvili et al., 2015b) was used to construct a complex of $\mathrm{hDAT}^{\mathrm{dDAT}}$ bound to 5-MAPB for subsequent atomistic MD simulations. The $\mathrm{hDAT}^{\mathrm{dDAT}}$ protein immersed in the membrane environment, in a box of explicit water, along with internal ions and added salts were preserved. The bound dopamine was swapped with 5MAPB to create the corresponding complex. To this end, the backbone atoms $\mathrm{N}, \mathrm{C} \square, \mathrm{C} \square$ and $\mathrm{C} 1$ of the benzofuran ring in 5-MAPB structure were aligned on the backbone atoms $\mathrm{N}$, $\mathrm{C} \square, \mathrm{C} \square$ and $\mathrm{C} 1$ of the catechol ring of dopamine to overlap the binding position of 5-MAPB to dopamine in $\mathrm{hDAT}^{\mathrm{dDAT}}$. After a short $(0.5 \mathrm{~ns})$ equilibration, this new system was subjected to unbiased MD simulations totalling 250ns. The simulations were carried out as described in (Khelashvili et al., 2015b) using ACEMD software (Harvey et al., 2009). Briefly, the simulations employed the all-atom CHARMM27 force field for proteins with CMAP corrections was used (Brooks et al., 2009) as well as the CHARMM36 force field for lipids (Klauda et al., 2010), the TIP3P water model, and the CHARMM-compatible force-field parameter set for $\mathrm{PIP}_{2}$ lipids (Lupyan et al., 2010). The PME method for electrostatic calculations was used, along with 4 fs integration time-step with standard mass repartitioning procedure for hydrogen atoms implemented in ACEMD. More details about the computational protocol can be found in (Khelashvili et al., 2015b).

The trajectory was analysed with VMD (Humphrey et al., 1996) and various analyses tools developed in-house. 
2.9 Statistics. For the binding study we analysed the data with a 1-way ANOVA. For the voltammetry study statistical analyses were performed on peak effects of each drug concentration on each brain slice, which was typically found around 45-50 min after drug application. Statistical analysis was carried out using SigmaPlot (v. 11.0). We used a 1-way ANOVA (independent variable $=$ concentration) with post-hoc Tukey's. In all graphs data are presented as means \pm SEMs and significance is set at $\mathrm{p}<0.05$. 


\section{Results}

Ligand Binding. 5-MAPB caused a concentration dependent displacement of $\left[{ }^{125}\right.$ I]RTI-121 from the DAT $(\mathrm{F}(4,29)=13.55, \mathrm{p}<0.001)$. Post hoc analysis with Dunnett's methods revealed that both 10 and $30 \mu \mathrm{M} 5$-MAPB caused a significant reduction, by $30 \%$ and $50 \%$ respectively, in $\left[{ }^{125} \mathrm{I}\right] \mathrm{RTI}-121$ binding versus the control $(\mathrm{p}<0.05$; Figure 2A-C); the cocaine/[ ${ }^{125}$ I]RTI-121 displacement curve shown for comparison (Figure 2D).

[Figure 2 here]

\section{Fast cyclic voltammetry in accumbens slices.}

There was evidence of reverse transport where application of 5-MAPB caused an increase in background dopamine levels independent of electrical stimulation. This was seen with 5MAPB at both 1 and $10 \mu \mathrm{M}$, although did not happen on every occasion (see Figure 3). 5MAPB also had an effect on electrically evoked dopamine efflux $(\mathrm{H}(3)=8.823, \mathrm{p}<0.05)$, post hoc analysis revealed that both 0.1 and $10 \mu \mathrm{M}$ 5-MAPB increased the AUC (Figure 4). We have previously shown that more classical DAT blockers such as cocaine diethylpropion and bupropion do not increase basal levels of dopamine, in contrast to amphetamine (OpackaJuffry et al., 2014).

[Figures 3 and 4 here]

Molecular modelling and docking of ligands. We built the molecular models for the binding of 5-MAPB, dopamine, amphetamine, 5-APB, MDMA, cocaine and RTI-121 in Rattus norvegicus dopamine transporter (rDAT) (Figure 5A and Supplemental Figure 2SA). These models were based on the high-resolution structure of the Drosophila melanogaster dopamine transporter, dDAT, bound to the neurotransmitter dopamine (PDB ID: 4XP1) (Wang et al., 2015) and a refined structure-based sequence alignment of neurotransmitter 
sodium symporter (NSS) proteins (Beuming et al., 2006). The docking results confirmed that the 5-MAPB pose overlaps with the dopamine, amphetamine, 5-APB, MDMA, cocaine and RTI-121 binding sites (Supplemental Figure 2SB). Indeed, comparison of molecular models of dopamine (Figure 5B), amphetamine (Figure 5C), 5-APB (Figure 5D), MDMA (Figure 5E), 5-MAPB (after an induced-fit protocol, that allows backbone flexibility, Figure 5F), cocaine (Supplemental Figure 2SC) and RTI-121 (Supplemental Figure 2SD) show that these compounds share a binding site at the centre of rDAT and located in a position corresponding to the primary substrate-binding site cavity (S1) seen in previous modelling studies in hDAT (Beuming et al., 2008; Bisgaard et al., 2011). Specifically, in rDAT, aromatic-aromatic stacking interactions are observed between Tyr156 and the catechol ring of dopamine, phenyl ring of amphetamine and the fused benzene and furan rings of 5-APB, 5-MAPB and MDMA. According to our initial models when dopamine (Figure 5B), amphetamine (Figure 5C), 5APB (Figure 5D), MDMA (Figure 5E), cocaine (Figure 2SC) and RTI-121 (Figure 2SD) are bound to rDAT $^{\mathrm{dDAT}}$ they do not disrupt the stabilizing hydrogen bond between Asp79 and Tyr156 shown to be important in stabilizing the S1 binding pocket (Beuming et al., 2008). The dock of 5-MAPB (Figure 5F) also suggests a hydrogen bond between the OH group of Tyr156 and Asp79 as well as a stabilising salt bridge between Asp79 and the protonated amine of 5-MAPB (Figure 5F), this similar motif has been described previously for dopamine, amphetamine and MDMA binding to hDAT (Beuming et al., 2008). These observations are similar to those reported from the recent dDAT crystal structures (Wang et al., 2015). In fact all reported poses of these docked compounds preserve $\sim 3 \AA$ - $4 \AA$ separation between Y156 and D79.

These findings are interesting, especially for the larger compounds, cocaine and RTI-121, when compared to previous modelling studies and the recent crystallographic data. A previous work (Beuming et al., Nature 2008) on a homology model of human DAT based on 
LeuT (Leucine transporter, bacterial homologue of NSS) bound to various small molecules including dopamine, amphetamine, benzotropine, the benzotropine analog JHW007, MDMA, cocaine, CFT explored the time-dependent behaviour of the Tyr-Asp hydrogen bond with molecular dynamics (MD) simulations. These studies concluded that larger molecules such as CFT and cocaine disrupt the Y156-D79 hydrogen bond; a finding that was supported by mutagenesis studies where dopamine uptake was markedly decreased because of the destabilization of the dopamine-binding pocket. In contrast, the paper by Wang et al., Nature 2015; captures structures of Drosophila Melongaster bound to cocaine, $\beta$-CFT and RTI-55 in an outward-open conformation and where the Asp46-Tyr124 (dDAT numbering) interaction is not disrupted similar to our initial docks. It is important to note, as mentioned above, that the previous model of hDAT was based on a template of LeuT (Beuming et al., 2008) while our current model of rDAT is based on dDAT, and includes both $\mathrm{N}$ - and $\mathrm{C}$-terminal regions, constructed from a previous protocol developed for hDAT (Khelashvili et al., 2015a).

While reconciliation of structural differences between different sized compounds are outside the context and scope of the current manuscript, we probed with atomistic MD simulations structural and dynamics characteristic of hDAT bound to 5-MAPB. Human DAT was chosen in order to facilitate comparison of the computational results with previous large-scale MD simulations of dopamine-bound hDAT (Khelashvili et al, 2015b). Thus, we have reported on spontaneous occluded-to-inward-facing transitions in hDAT and concomitant release of $\mathrm{Na}+$ ion from the functional $\mathrm{Na} 2$ site $\left(\mathrm{Na} 2\right.$ ion) induced by $\mathrm{PIP}_{2}$ lipid-mediated interactions between the $\mathrm{N}$-terminal domain and the intracellular loop 4 (IL4) of hDAT. Here, starting with the occluded model of hDAT (with $\mathrm{Na}+$ and $\mathrm{Cl}$ - ions in their respective binding sites) but with substituting dopamine in the S1 site with 5-MAPB (see Methods), we observed similar destabilization of the $\mathrm{Na} 2$ ion (Figure 6A) and isomerization to the inward-facing 
state (as monitored by the disruption of the intracellular network of ionic interactions including R60-D436 and Y335-R428, Figure 6B) during 250ns atomistic MD simulations.

Interestingly, we find that the Y156-D79 minimum distance is maintained at $\sim 4 \AA$ throughout the trajectory decreasing to even smaller $(\sim 2 \AA)$ distances towards the end of the simulation (Figure 6A) suggesting that Y156-D79 interaction is preserved in the presence of 5-MAPB. These computational studies highlight that when in complex with 5-MAPB, DAT can exhibit conformational dynamics that lead to spontaneous isomerization of the transporter into inward-facing state, similarly to that observed in dopamine-bound DAT (Khelashvili et al, 2015b).

Figure 5 and Figure 6 here

\section{Discussion}

We characterised a stimulant profile of an exemplar benzofuran NPS by means of a set of methods, that included not only the previously used neurobiological approaches, autoradiography and in vitro fast cyclic voltammetry, but also a biophysical method of molecular modelling of interactions between ligands and their biological targets.

We chose 5-MAPB as an example of NPS and yet nearly unknown for its pharmacological properties (Welter-Luedeke and Maurer, 2015). There are no previous studies of the pharmacology of 5-MAPB, but user reports suggest that this benzofuran is a stimulant and empathogen/entactogen, as well as having some hallucinogenic properties. The benzofurans were initially marketed as a legal form of MDMA (Ecstasy); the UK Advisory Council on the Misuse of Drugs (ACMD) considers 5-MAPB to be structurally closer to MDMA (See Figure 5A) than 5-APB, see also (Welter et al., 2015a, 2015b). We have previously examined the neuropharmacology of 5-APB and shown that it displaces RTI-121 from the DAT and 
ketanserin from the 5- $\mathrm{HT}_{2 \mathrm{~A}}$ receptor; it also causes a prolongation of electrically evoked dopamine (Dawson et al., 2014).

The present data demonstrate that 5-MAPB binds to the DAT in rat brain tissue, where it competes at the transporter with the selective DAT ligand, $\left[{ }^{125} \mathrm{I}\right] \mathrm{RTI}-121$, in a concentrationdependent manner, but being less potent than cocaine. In that it resembles 5-APB (Dawson et al., 2014). The autoradiography analysis, while of high anatomical resolution and fully quantitative to assess ligand competition in bran tissue in vitro, does not inform about the very mechanism of interaction between the drug tested and the DAT, in particular it does not distinguish whether the drug competitively blocks the DAT and therefore reduces dopamine reuptake, largely like cocaine e.g. (Jones et al., 1995) or whether the drug interacts with DAT to enter the presynaptic compartment where it displaces newly synthesised dopamine the way amphetamine does (Butcher et al., 1988).

In this respect, the functional voltammetry approach complements the binding study as it can distinguish between the states of dopamine reuptake inhibition versus enhanced dopamine release. It achieves that through the real time (sub-second) analysis of dopamine efflux following electrical stimulation before-and-after a drug application, as we have demonstrated for a range of cathinones (Opacka-Juffry et al., 2014). The present study revealed that 5MAPB can cause reverse transport and also increases the AUC of electrically evoked dopamine efflux. These data are consistent with 5-MAPB having a stimulant profile.

Interactions between stimulant NPS and DAT may be even more complex as there is evidence that cocaine, formerly seen as a classical DAT blocker, can not only block dopamine reuptake but also amplify dopamine release by mobilizing a reserve pool of dopamine-containing synaptic vesicles (Venton et al., 2006). The intricate mechanisms of stimulant-DAT interactions, which lead to augmentation of dopamine neurotransmission, 
should be investigated and understood in the future as they are important for the psychostimulant effects and addictive potential of drugs including NPS. Such studies of molecular interactions are theoretically achievable by means of biophysical methods of molecular modelling; our present approach is a stepping stone in that direction.

The present molecular modelling and docking studies reveal a binding mode for 5-MAPB that is found central to the transmembrane domains $1,3,6$ and 8 , which overlaps extensively with that of dopamine, amphetamine, 5-APB, MDMA, cocaine and its analogues. We observe the conservation of the Tyr156-Asp79 hydrogen bond when dopamine, amphetamine, 5-MAPB, 5-APB, MDMA, cocaine and RTI-121 are docked to the rDAT $^{\mathrm{dDAT}}$ model. Although the present dock of 5-MAPB suggests a hydrogen bond between Tyr156 and Asp79 similar to dopamine, amphetamine, 5-APB, MDMA, cocaine and RTI-121 seen from the recent crystal structure of dDAT (Wang et al., 2015), the stability and timedependent behaviour of these interactions need to be quantitatively investigated with molecular dynamics simulations to explain the differences seen between the previous models based on LeuT (Beuming et al., 2008) and the recent crystal structures (Wang et al., 2015). Atomistic MD simulations performed here to investigate the conformational dynamics in DAT when complexed with 5-MAPB revealed the spontaneous release of $\mathrm{Na}+$ ion from the $\mathrm{Na} 2$ site as well as disruption of the intracellular network of ionic interactions that stabilize DAT in the inward-closed state (Kniazeff et al., 2008) suggesting that the 5-MAPB-bound transporter exhibits conformational changes that are consistent with the early stages of inward opening, as observed for dopamine-bound DAT (Khelashvili et al, 2015b).

Longer simulations are needed to observe the effect of different sized ligands on the structural rearrangements of the binding site residues, such as investigating the occurrence of the stabilising interactions of the $\mathrm{OH}$ group of Tyr156 and Asp79 and the salt bridge between 
Asp79 and the protonated amine of 5-MAPB and binding affinity for structurally similar compounds that may differ in functional groups or moieties. Free-energy calculations can be further implemented to address these questions after a thorough understanding of the dynamics of the system gathered from sufficiently long unbiased molecular dynamics.

Conformational changes emerging over long-scale simulations can indicate the structural and dynamic elements of the mechanisms governing the interactions of 5-MAPB with DAT. This work is ongoing but inferences can then be probed using comparisons among various structural families of ligands, which are expected to elicit structure-related structural and dynamic consequences of binding.

A mechanistic characterization of these changes in rat DAT is of direct relevance as this is the primary animal model used in functional studies and pre-clinical drug testing. A structural comparison is needed to determine the relevance of binding affinity data from rat models used in translational studies that ultimately inform decisions on drug classification. In this respect, it is important to establish if functional phenotypes of 5-MAPB and amphetamine can be explained by common mechanisms. Indeed, extensive studies of amphetamineinduced and DAT-mediated efflux of dopamine have established that the efflux process requires activation of specific molecular machinery in the cell that includes various kinases, scaffolding proteins, and even specific lipid membrane components acting on DAT (Khelashvili et al., 2015b; Khelashvili and Weinstein, 2015). It, therefore, remains to be seen whether 5-MAPB-induced dopamine efflux as well is dependent on activation of similar molecular machinery.

We are further developing molecular modelling approaches to study NPS stimulant effects, with dynamic analysis of various compounds and their analogues interacting with the monoamine transporters. With the determination of the new crystal structure of hSERT (Coleman et al., 2016), investigations of a large repertoire of stimulant compounds bound to 
hSERT, hDAT and hNET could provide a pharmacological characterisation of selectivity when combined with functional studies. Such novel molecular-computational approaches, working in concert with traditional functional studies to define the stimulant properties of NPS and effectively their addictive potential, are advantageous and should become increasingly applicable in the future.

The present data provide the first pharmacological and structural evidence of 5-MAPB's stimulant mechanism of action, and suggest potential addictive properties, which informs about the health risk related to its use. The present study demonstrates the benefits of combining computational methods of biophysics with neurobiological procedures by providing novel insights into the structural determinants of NPS at the dopamine transporter and subsequently reconciling them with their functional properties at DAT as the main molecular target of stimulant drugs of addiction.

\section{Conclusions}

We have found 5-MAPB to bind to the DAT and displace RTI-121 as DAT ligand, and to slow down reuptake of electrically evoked dopamine in the rat accumbens. In addition 5MAPB can cause reverse transport of dopamine, which is a feature of amphetamine-like substances.

Molecular modelling has demonstrated a binding mode for 5-MAPB at DAT that occupies a binding pocket that is deeply buried in the transporter protein structure and overlaps with the binding site of the substrate dopamine and other stimulants such as amphetamine, MDMA, 5APB, cocaine and RTI-121. Atomistic simulations indicate a number of structural rearrangements that stabilise the inward facing conformation of DAT when bound to 5MAPB, such as the spontaneous release of $\mathrm{Na} 2$ and the rearrangement of the intracellular gates. These findings support the functional neurochemistry findings of the initial transport 
steps and longer atomistic simulations are needed to resolve whether the stimulant behaves like amphetamine.

The present data, by combining computational methods of biophysics with neurobiological procedures, provide the first structural and pharmacological evidence of 5-MAPB's stimulant mechanism of action, and suggest its potential addictive properties, which informs about the health risk related to its use. Such approaches can be applied to assess addictive potential of other NPS.

Acknowledgements. This study was funded by the European Commission Drug prevention and Information Program 2014-16, JUST/2013/DPIP/AG/4823, EU MADNESS project. The following computational resources are gratefully acknowledged: ARCHER granted via the UK High-End Computing Consortium for Biomolecular Simulation, HECBioSim (http://hecbiosim.ac.uk), supported by EPSRC (grant no. EP/L000253/1); and the computational resources of the David A. Cofrin Center for Biomedical Information in the HRH Prince Alwaleed Bin Talal Bin Abdulaziz Alsaud Institute for Computational Biomedicine, USA.

\section{Authors' contribution}

CD, MAS, HW and JOJ were responsible for the study concept and design. CD and VB collected and interpreted the voltammetry data. JOJ and ND conducted the ligand binding experiments and ND analysed the data. MAS and GK performed the molecular modelling studies and interpreted the findings. CD, MAS, GK and JOJ drafted the manuscript. All authors critically reviewed the content and approved the final version for publication. 


\section{References}

Beuming, T., Kniazeff, J., Bergmann, M.L., Shi, L., Gracia, L., Raniszewska, K., Newman, A.H., Javitch, J.A., Weinstein, H., Gether, U., Loland, C.J., 2008. The binding sites for cocaine and dopamine in the dopamine transporter overlap. Nat. Neurosci. 11, 780-9.

Beuming, T., Shi, L., Javitch, J.A., Weinstein, H., 2006. A comprehensive structure-based alignment of prokaryotic and eukaryotic neurotransmitter/Na+ symporters (NSS) aids in the use of the LeuT structure to probe NSS structure and function. Mol. Pharmacol. 70, $1630-42$.

Bisgaard, H., Larsen, M.A.B., Mazier, S., Beuming, T., Newman, A.H., Weinstein, H., Shi, L., Loland, C.J., Gether, U., 2011. The binding sites for benztropines and dopamine in the dopamine transporter overlap. Neuropharmacology 60, 182-90.

Brooks, B.R., Brooks, C.L., Mackerell, A.D., Nilsson, L., Petrella, R.J., Roux, B., Won, Y., Archontis, G., Bartels, C., Boresch, S., Caflisch, A., Caves, L., Cui, Q., Dinner, A.R., Feig, M., Fischer, S., Gao, J., Hodoscek, M., Im, W., Kuczera, K., Lazaridis, T., Ma, J., Ovchinnikov, V., Paci, E., Pastor, R.W., Post, C.B., Pu, J.Z., Schaefer, M., Tidor, B., Venable, R.M., Woodcock, H.L., Wu, X., Yang, W., York, D.M., Karplus, M., 2009. CHARMM: the biomolecular simulation program. J. Comput. Chem. 30, 1545-614.

Butcher, S.P., Fairbrother, I.S., Kelly, J.S., Arbuthnott, G.W., 1988. Amphetamine-induced dopamine release in the rat striatum: an in vivo microdialysis study. J. Neurochem. 50, $346-355$.

Coleman, J.A., Green, E.M., Gouaux, E., 2016. X-ray structures and mechanism of the human serotonin transporter. Nature 532, 334-339.

Dawson, P., Opacka-Juffry, J., Moffatt, J.D., Daniju, Y., Dutta, N., Ramsey, J., Davidson, C., 
2014. The effects of benzofury (5-APB) on the dopamine transporter and 5-HT2dependent vasoconstriction in the rat. Prog. Neuropsychopharmacol. Biol. Psychiatry 48, $57-63$.

Di Chiara, G., Bassareo, V., 2007. Reward system and addiction: what dopamine does and doesn’t do. Curr. Opin. Pharmacol.

Di Chiara, G., Imperato, A., 1988. Drugs abused by humans preferentially increase synaptic dopamine concentrations in the mesolimbic system of freely moving rats. Proc. Natl. Acad. Sci. U. S. A. 85, 5274-5278.

Hamilton, P.J., Campbell, N.G., Sharma, S., Erreger, K., Herborg Hansen, F., Saunders, C., Belovich, A.N., Sahai, M.A., Cook, E.H., Gether, U., McHaourab, H.S., Matthies, H.J.G., Sutcliffe, J.S., Galli, A., 2013. De novo mutation in the dopamine transporter gene associates dopamine dysfunction with autism spectrum disorder. Mol. Psychiatry $18,1315-23$.

Hansen, F.H., Skjørringe, T., Yasmeen, S., Arends, N. V, Sahai, M.A., Erreger, K., Andreassen, T.F., Holy, M., Hamilton, P.J., Neergheen, V., Karlsborg, M., Newman, A.H., Pope, S., Heales, S.J.R., Friberg, L., Law, I., Pinborg, L.H., Sitte, H.H., Loland, C., Shi, L., Weinstein, H., Galli, A., Hjermind, L.E., Møller, L.B., Gether, U., 2014. Missense dopamine transporter mutations associate with adult parkinsonism and ADHD. J. Clin. Invest. 124, 3107-20.

Harvey, M.J., Giupponi, G., Fabritiis, G. De, 2009. ACEMD: Accelerating Biomolecular Dynamics in the Microsecond Time Scale. J. Chem. Theory Comput. 5, 1632-1639. Hume, S.P., Luthra, S.K., Brown, D.J., Opacka-Juffry, J., Osman, S., Ashworth, S., Myers, R., Brady, F., Carroll, F.I., Kuhar, M.J., Brooks, D.J., 1996. Evaluation of [11C]RTI- 
121 as a selective radioligand for PET studies of the dopamine transporter. Nucl. Med. Biol.

Humphrey, W., Dalke, A., Schulten, K., 1996. VMD-Visual Molecular Dynamics. J MolGraphics 14, 33-38.

Iversen, L., Gibbons, S., Treble, R., Setola, V., Huang, X.P., Roth, B.L., 2013.

Neurochemical profiles of some novel psychoactive substances. Eur. J. Pharmacol. 700, $147-151$.

Jones, S.R., Garris, P. a, Wightman, R.M., 1995. Different effects of cocaine and nomifensine on dopamine uptake in the caudate-putamen and nucleus accumbens. J. Pharmacol. Exp. Ther. 274, 396-403.

Khelashvili, G., Doktorova, M., Sahai, M.A., Johner, N., Shi, L., Weinstein, H., $2015 a$. Computational modeling of the $\mathrm{N}$-terminus of the human dopamine transporter and its interaction with PIP 2 -containing membranes. Proteins Struct. Funct. Bioinforma. 83, 952-969.

Khelashvili, G., Stanley, N., Sahai, M.A., Medina, J., LeVine, M. V, Shi, L., De Fabritiis, G., Weinstein, H., 2015b. Spontaneous Inward Opening of the Dopamine Transporter Is Triggered by PIP2-Regulated Dynamics of the N-Terminus. ACS Chem. Neurosci. 6, $1825-37$.

Khelashvili, G., Weinstein, H., 2015. Functional mechanisms of neurotransmitter transporters regulated by lipid-protein interactions of their terminal loops. Biochim. Biophys. Acta $1848,1765-74$.

Klauda, J.B., Venable, R.M., Freites, J.A., O’Connor, J.W., Tobias, D.J., MondragonRamirez, C., Vorobyov, I., MacKerell, A.D., Pastor, R.W., 2010. Update of the 
CHARMM All-Atom Additive Force Field for Lipids: Validation on Six Lipid Types. J. Phys. Chem. B 114, 7830-7843.

Kniazeff, J., Shi, L., Loland, C.J., Javitch, J.A., Weinstein, H., Gether, U., 2008. An intracellular interaction network regulates conformational transitions in the dopamine transporter. J. Biol. Chem. 283, 17691-701.

Kuhar, M.J., Ritz, M.C., Boja, J.W., 1991. The dopamine hypothesis of the reinforcing properties of cocaine. Trends Neurosci. 14, 299-302.

Lee, T.H., Gee, K.R., Davidson, C., Ellinwood, E.H., 2002. Direct, real-time assessment of dopamine release autoinhibition in the rat caudate-putamen. Neuroscience 112, 647654.

Lupyan, D., Mezei, M., Logothetis, D.E., Osman, R., 2010. A molecular dynamics investigation of lipid bilayer perturbation by PIP2. Biophys. J. 98, 240-7.

Monte, A.P., Marona-Lewicka, D., Cozzi, N. V., Nichols, D.E., 1993. Synthesis and pharmacological examination of benzofuran, indan, and tetralin analogues of 3,4(methylenedioxy)amphetamine. J Med Chem 36, 3700-3706.

np_SAD report, 2014. http://www.sgul.ac.uk/images/NPSAD_-_Drugrelated_deaths_in_England_Northern_Ireland_the_Channel_Islands_and_the_Isle_of_M an_January-December_2013.pdf.

Opacka-Juffry, J., Pinnell, T., Patel, N., Bevan, M., Meintel, M., Davidson, C., 2014. Stimulant mechanisms of cathinones - effects of mephedrone and other cathinones on basal and electrically evoked dopamine efflux in rat accumbens brain slices. Prog. Neuropsychopharmacol. Biol. Psychiatry 54, 122-30. 
Paxinos, G Watson, C., 2007. The rat brain in stereotaxic coordinates. Elsevier.

Phillips, P.E.M., Hancock, P.J., Stamford, J.A., 2002. Time window of autoreceptor-mediated inhibition of limbic and striatal dopamine release. Synapse 44, 15-22.

Rickli, A., Kopf, S., Hoener, M.C., Liechti, M.E., 2015. Pharmacological profile of novel psychoactive benzofurans. Br. J. Pharmacol. 172, 3412-25.

Robinson, D.L., Venton, B.J., Heien, M.L.A. V, Wightman, R.M., 2003. Detecting subsecond dopamine release with fast-scan cyclic voltammetry in vivo, in: Clinical Chemistry. American Association for Clinical Chemistry, pp. 1763-1773.

Schrödinger, LLC: Portland, OR, 2007, Web address: www.schrodinger.com. [WWW Document], n.d. URL www.schrodinger.com

Shimshoni, J.A., Winkler, I., Golan E., Nutt D., 2016. Neurochemical binding profiles of novel indole and benzofuran MDMA analogues. Naunyn Schmiedebergs Arch Pharmacol. 2016 Sep 20. [Epub ahead of print]

Strazielle, C., Lalonde, R., Amdiss, F., Botez, M.I., Hébert, C., Reader, T.A., 1998. Distribution of dopamine transporters in basal ganglia of cerebellar ataxic mice by [125I]RTI-121 quantitative autoradiography. Neurochem. Int. 32, 61-68.

The Misuse of Drugs Act 1971 (Ketamine etc.) (Amendment) Order 2014, n.d. . Queen’s Printer of Acts of Parliament.

Venton, B.J., Seipel, A.T., Phillips, P.E., Wetsel, W.C., Gitler, D., Greengard, P., Augustine, G.J., Wightman, R.M., 2006. Cocaine increases dopamine release by mobilization of a synapsin-dependent reserve pool. J Neurosci 26, 3206-3209.

Wang, K.H., Penmatsa, A., Gouaux, E., 2015. Neurotransmitter and psychostimulant 
recognition by the dopamine transporter. Nature 521, 322-7.

Welter-Luedeke, J., Maurer, H.H., 2015. New Psychoactive Substances: Chemistry, Pharmacology, Metabolism, and Detectability of Amphetamine Derivatives with Modified Ring Systems. Ther. Drug Monit. 38, 4-11.

Welter, J., Brandt, S.D., Kavanagh, P., Meyer, M.R., Maurer, H.H., 2015a. Metabolic fate, mass spectral fragmentation, detectability, and differentiation in urine of the benzofuran designer drugs 6-APB and 6-MAPB in comparison to their 5-isomers using GC-MS and LC-(HR)-MSn techniques. Anal. Bioanal. Chem. 407, 3457-3470.

Welter, J., Kavanagh, P., Meyer, M.R., Maurer, H.H., 2015b. Benzofuran analogues of amphetamine and methamphetamine: Studies on the metabolism and toxicological analysis of 5-APB and 5-MAPB in urine and plasma using GC-MS and LC-(HR)-MSn techniques. Anal. Bioanal. Chem. 407, 1371-1388.

Yorgason, J.T., España, R.A., Jones, S.R., 2011. Demon Voltammetry and Analysis software: Analysis of cocaine-induced alterations in dopamine signaling using multiple kinetic measures. J. Neurosci. Methods 202, 158-164. 


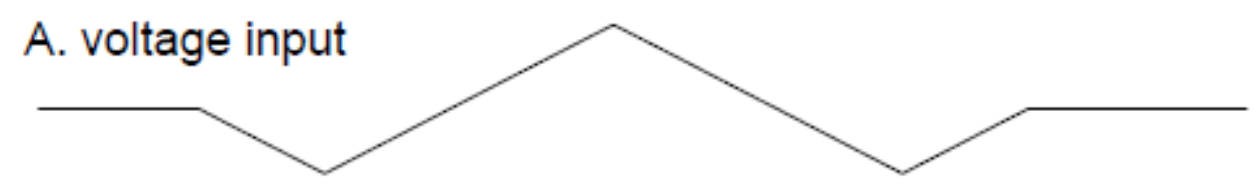

\section{B. current at electrode}
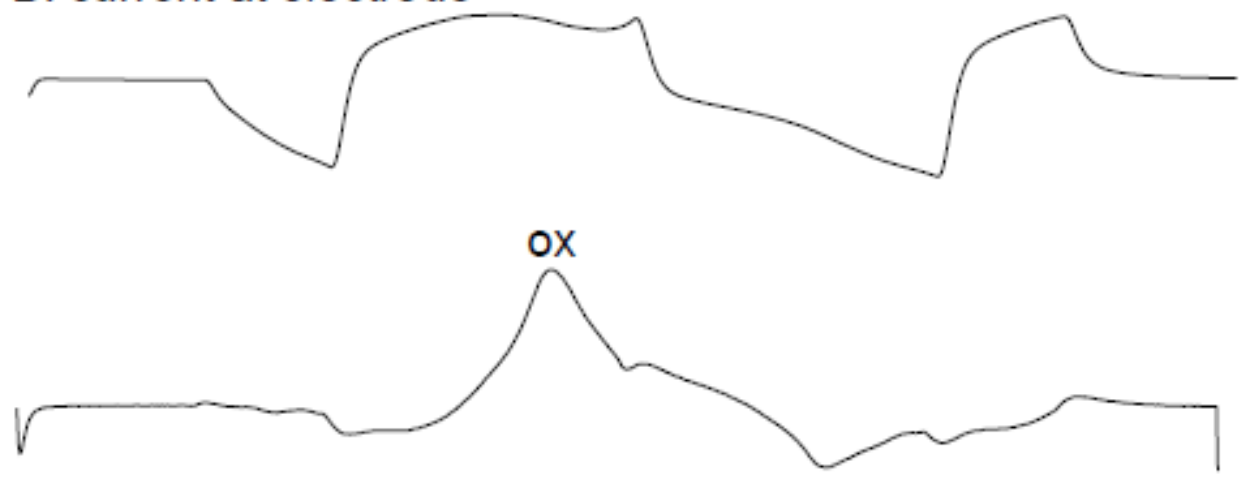

\section{C. subtracted Faradaic current}

red

\section{Figure 1. Methods for voltammetry showing 5-MAPB causes reverse transport of}

dopamine. A: input voltage waveform applied to the carbon fibre electrode ( 0 to -1 to +1.4 to -1 and back to $0 \mathrm{~V}$ at $480 \mathrm{~V} / \mathrm{s}$ ). B: resultant charging current at the carbon fibre electrode. $\mathrm{C}$ : if one takes the electrode current in the presence of dopamine and subtracts the signal in the absence of dopamine one should be left with only a Faradaic current from the oxidation and reduction of dopamine. Here we see an oxidation peak at $\sim 600 \mathrm{mV}$ and a reduction peak at $200 \mathrm{mV}$, exactly where one would expect to see peaks if dopamine was present. The data from ' $C$ ' are taken from Figure $3 B$ and the signal at ' $Y$ ' is subtracted from the signal at ' $X$ '. These data show that 5-MAPB can cause reverse transport of dopamine. 

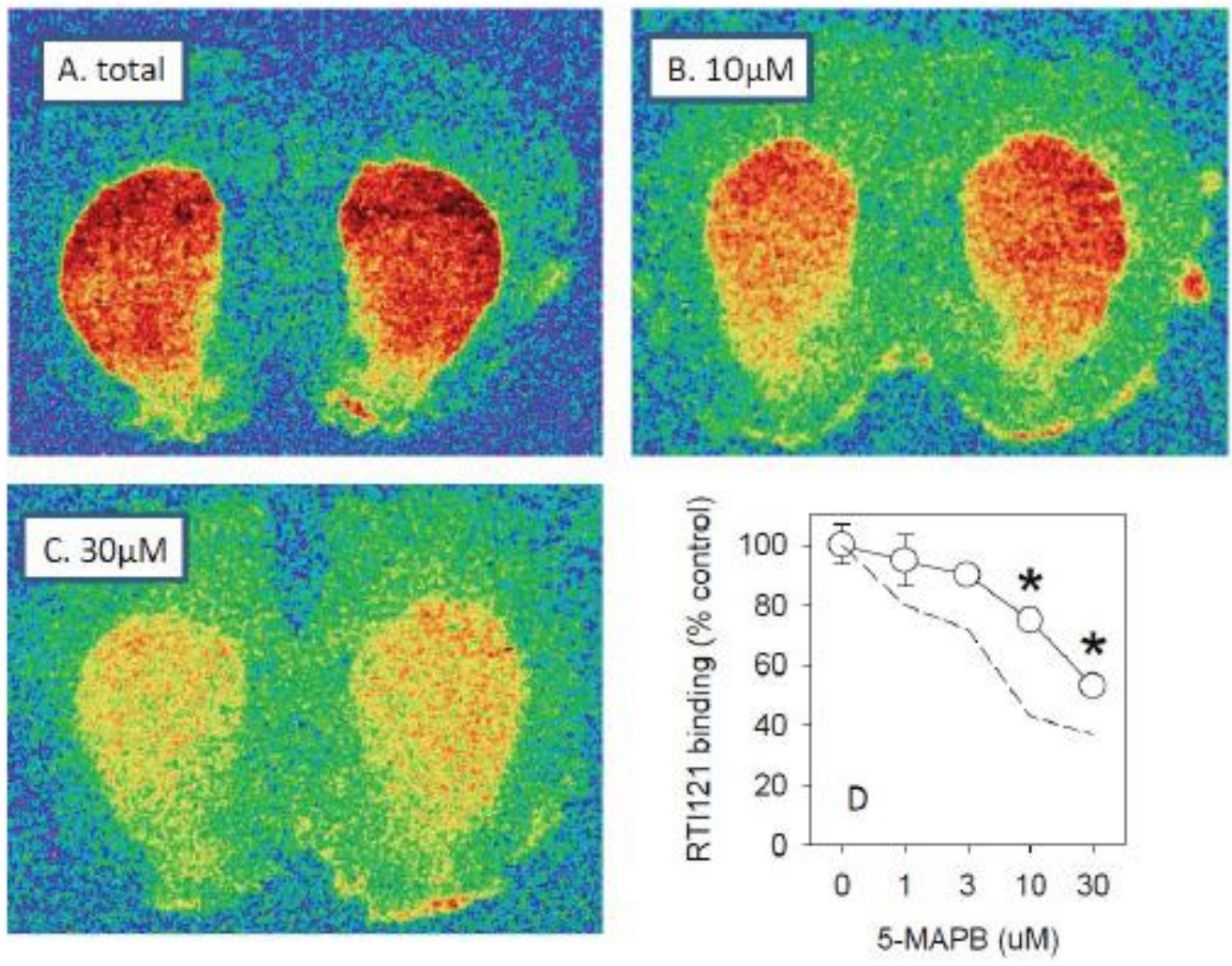

Figure 2. Displacement of [125I]RTI-121 by 5-MAPB in rat caudate sections. 5-MAPB displaced [125I]RTI-121-in a concentration dependent manner; (A) total [125I]RTI-121 binding; binding of [125I]RTI-121 in the presence of (B) $10 \mu \mathrm{M} 5$-MAPB and (C) $30 \mu \mathrm{M} 5$ MAPB; (D) combined concentration response curve for displacement of [125I] RTI21 binding by 5-MAPB, data for cocaine are derived from Opacka-Juffry et al., (2014) and given for comparison (dashed-line). Values are means \pm SEM. $* \mathrm{P}<0.05$ versus control $(0 \mu \mathrm{M})$, $\mathrm{n}=6$ per drug concentration. 
A. no reverse transport

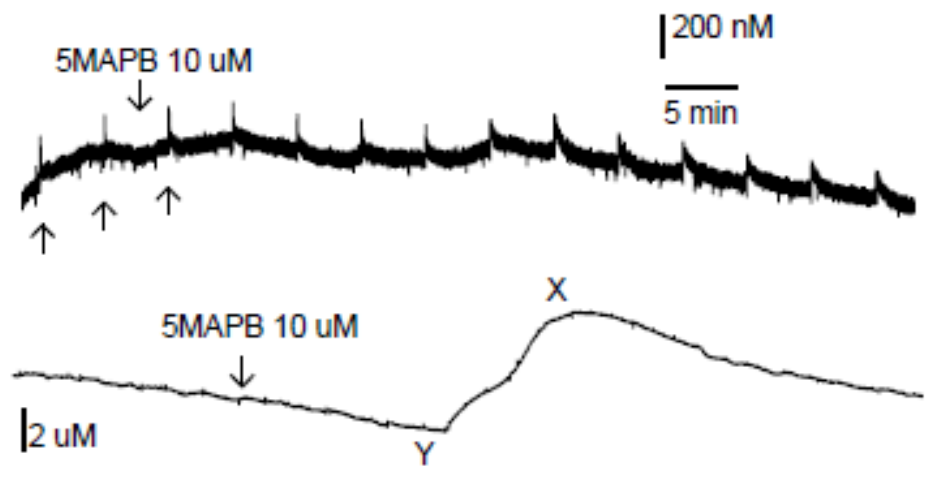

B. reverse transport

Figure 3. Dopamine efflux measured from the accumbens core. A. shows a typical experiment where we stimulate (10 pulses at $100 \mathrm{~Hz}$, upward arrows) every 5 min to evoke dopamine release. 5-MAPB $10 \mu \mathrm{M}$ is added at the downward arrow. One can see that the peak height of evoked dopamine does not increase, but towards the end of the experiment the dopamine efflux event gets longer suggesting that the DAT is blocked. B. is essentially the same experiment as ' $\mathrm{A}$ ' except that on this occasion when 5-MAPB, again at $10 \mu \mathrm{M}$, is added, one sees a large increase in the background signal, similar to what one might see with amphetamine. When the signal was electrochemically analysed one could see that the increase was due to dopamine (see Figure 1C). Note that the data on the upper panel are on a different vertical scale to the lower panel, by a factor of 10 . 

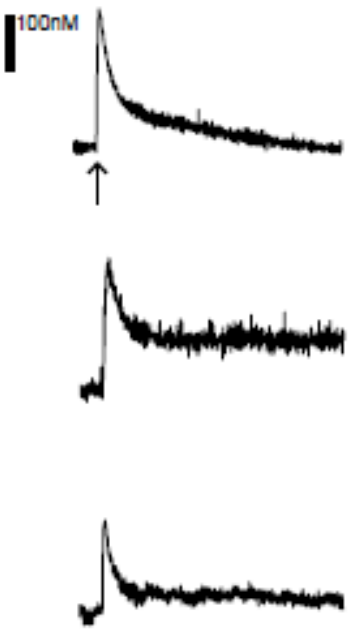

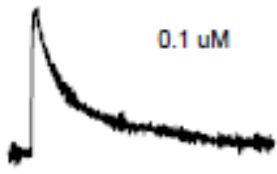

uM

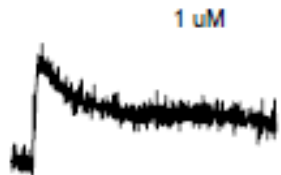

10 uM

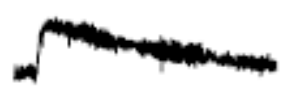

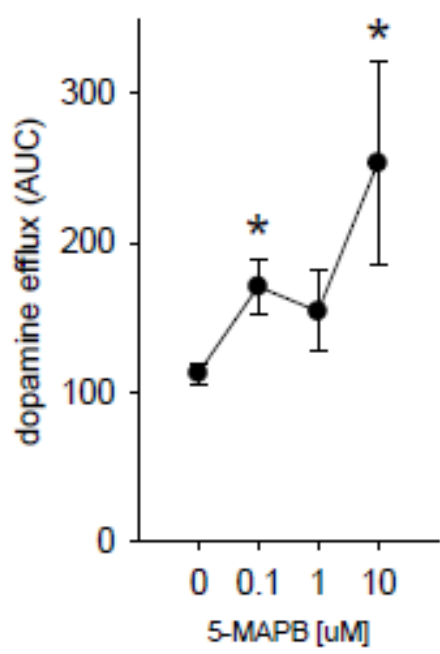

Figure 4. Effect of 5-MAPB on electrically evoked dopamine efflux. Left panels show raw data before (far left) and 50-60 min after (middle) 5-MAPB application. The upward arrow shows where electrical stimulation $(10$ pulses at $100 \mathrm{~Hz})$ took place. Note that peak dopamine efflux does not increase but reuptake is slowed after 5-MAPB. Right panel: concentration response curve showing that 5-MAPB at 0.1 and $10 \mu \mathrm{M}$ increase AUC dopamine efflux. *p < 0.05 . Values are means \pm SEM, $\mathrm{n}=4-6$. 
A.<smiles>CCCCCCCCC1CCC(OC)C(C)C1</smiles>

Doparnine<smiles>CC(N)CC1CC[C@H]2OCC[C@@H]2C1</smiles>

5-APB<smiles>COC(C)CC1CCC2CCCC2C1</smiles>

C.

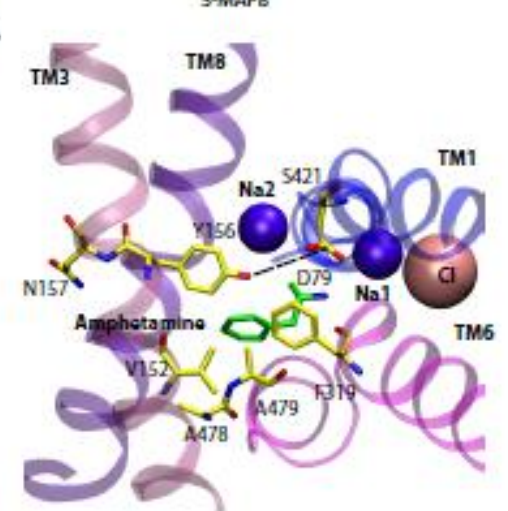

E.

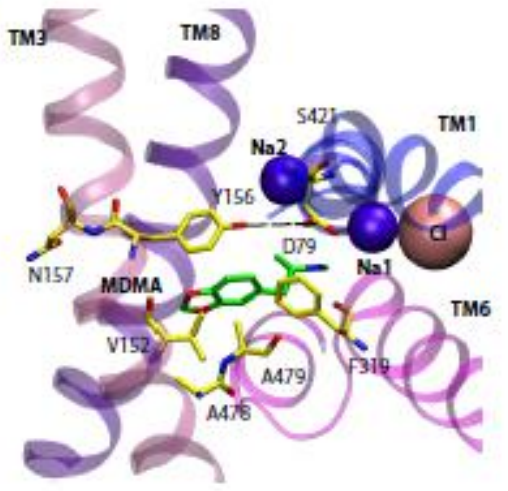

B.

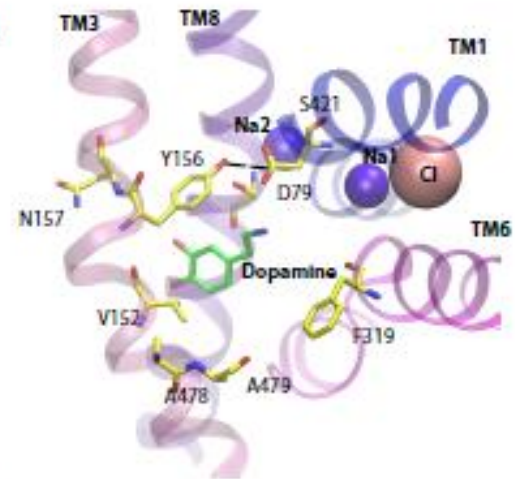

D.

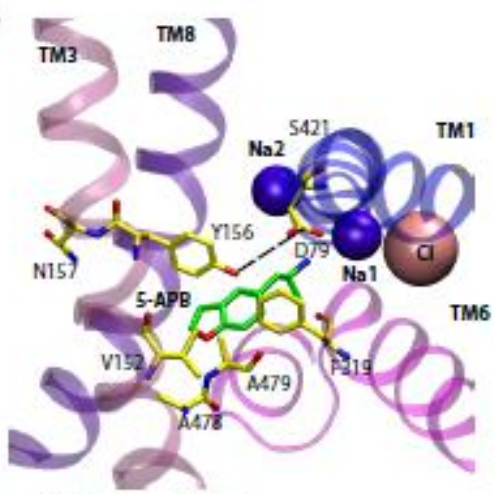

F.

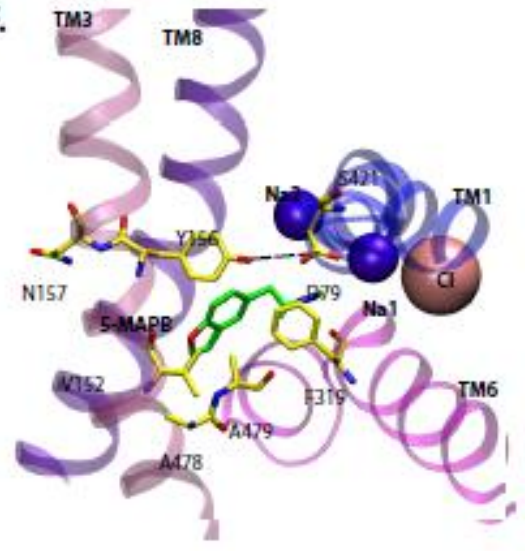

Figure 5. Molecular models of rDAT and ligands. (A) Structure of dopamine, amphetamine, 5-MAPB, MDMA and 5-APB. The represented poses as well as selected binding site residues are depicted individually in panels B to F. Molecular docking models of (B) dopamine, (C) amphetamine, (D) 5-APB (E) MDMA and (F) 5-MAPB (See Supplemental Figure 1S for details of docking pose). Each of these distinct ligands occupies a binding pocket that is deeply buried in the transporter structure and overlaps with the binding site of the substrate dopamine. Selected central binding site residues from each ligand are shown in yellow and labelled respectively. 

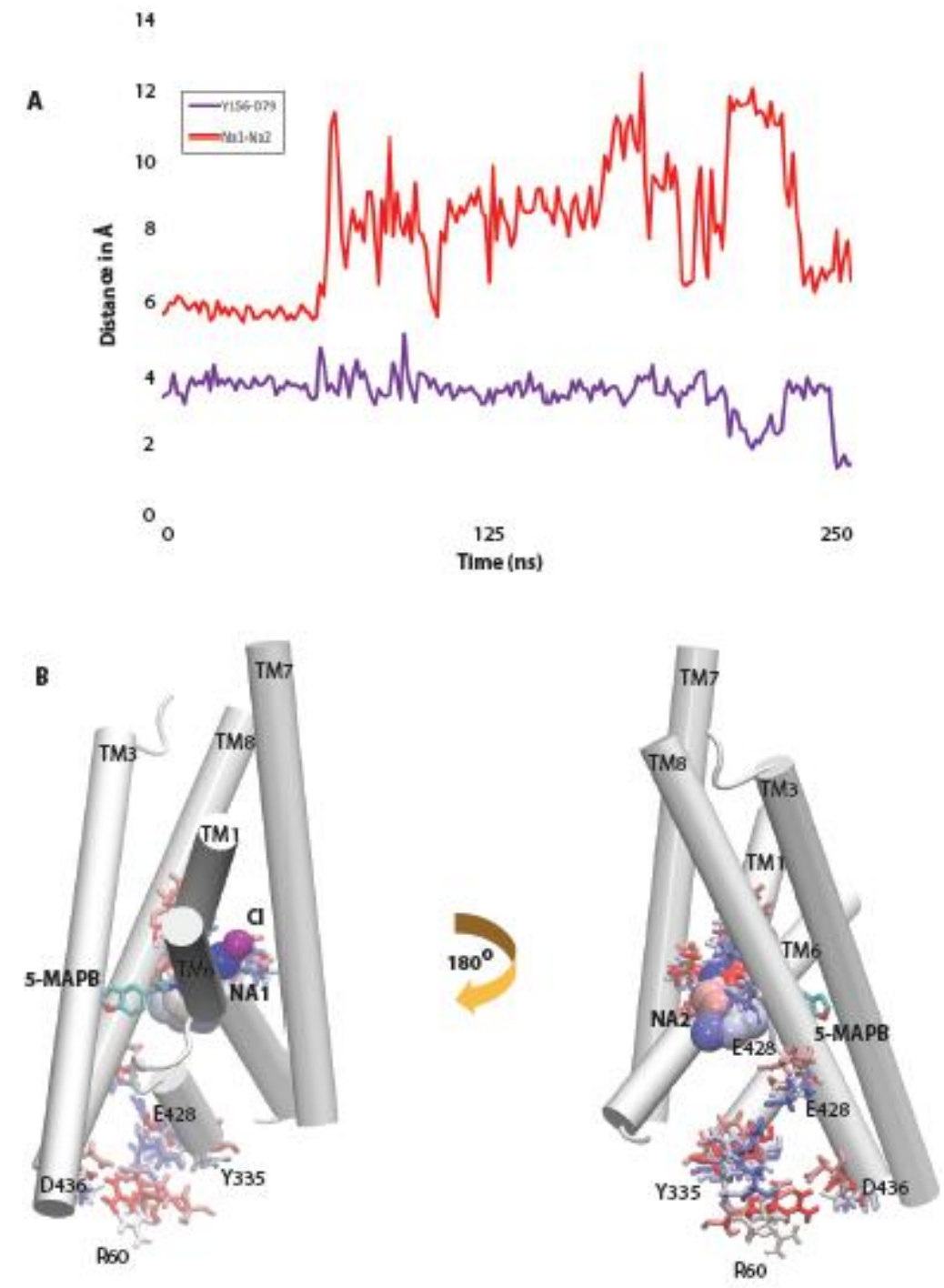

Figure 6. Spontaneous release of the $\mathrm{Na}+$ ion from the $\mathrm{Na} 2$ site in the 5-MAPB bound to hDAT $^{\text {dDAT }}$ (in white rendering) simulation. (A) Time-evolution, during the hDAT ${ }^{\mathrm{dDAT}}$ run, of minimum distances between Y156 and D79 (purple) and between the two Na+ ions initially in the $\mathrm{Na} 1$ and $\mathrm{Na} 2$ sites (red). (B) The positions of the ions and the ligand and relevant TM regions of $\mathrm{hDAT}^{\mathrm{dDAT}}$ are taken from the initial conformation of the system. The rearrangement of the various residues of the intracellular gates: R60, D436, E428 and Y335 and the residues coordinating Na2: L418, D421, G75 and V78 are rendered in licorice at intervals of 40 timesteps. $\mathrm{Na} 2$ is rendered in van der waals and its displacement from the $\mathrm{Na} 2$ site is represented also at intervals of 40 timesteps. $\mathrm{Na} 1$ and $\mathrm{Cl}$ ions are coloured in blue and pink, respectively, for clarity. The snapshots are related to each other by 180 o rotation around the vertical axis (direction perpendicular to the membrane plane). 
Highlights

Neurobiological and molecular modelling analyses indicate that 5-MAPB 5-MAPB binds to the dopamine transporter (DAT) and displaces RTI-121 as DAT ligand in rat brain tissue.

5-MAPB slows down reuptake of electrically-evoked dopamine in the rat accumbens and causes reverse transport of dopamine, which is a feature of amphetamine-like substances.

Molecular dynamics simulations describe the interactions between 5-MAPB and DAT. These approaches can be used for other NPS when assessing their functional properties at DAT as the molecular target of stimulants. 